\title{
Equivariant vector bundles over quantum projective spaces
}

\author{
A. Mudrov* \\ In memory of Ludwig Faddeev \\ Department of Mathematics, \\ University of Leicester, \\ University Road, LE1 7RH Leicester, UK
}

\begin{abstract}
We construct equivariant vector bundles over quantum projective spaces making use of parabolic Verma modules over the quantum general linear group. Using an alternative realization of the quantized coordinate ring of projective space as a subalgebra in the algebra of functions on the quantum group, we reformulate quantum vector bundles in terms of quantum symmetric pairs. In this way, we prove complete reducibility of modules over the corresponding coideal stabilizer subalgebras, via the quantum Frobenius reciprocity.
\end{abstract}

Key words: quantum groups, quantum projective spaces, vector bundles, symmetric pairs. AMS classification codes: 17B10, 17B37, 53D55.

*This study is supported by the RFBR grant 15-01-03148. 


\section{Introduction}

We quantize equivariant vector bundles over complex projective spaces regarded as one-sided projective modules over coordinate rings. It is a natural next step in the deformation quantization programme for Poisson manifolds, after quantization of their algebras of function, [BFFLS], considered as trivial bundles. In the presence of a symmetry group, quantization becomes a topic of representation theory.

The main idea underlying quantization is to realize the module of global sections of an associated vector bundle by a vector space of linear maps between certain highest weight modules over the classical/quantum total group. From this point of view the problem was addressed within the theory of dynamical twist in [DM1], for homogeneous spaces with Levi isotropy subgroups. It was later realized that trivial vector bundles (e. g., algebras of functions) admit a similar approach with regard to semi-simple conjugacy classes (consisting of semi-simple elements) with non-Levi isotropy subgroups, [M1, AM]. Those findings suggest a uniform quantization scheme for associated vector bundles over all semi-simple conjugacy classes, making use of highest weight modules.

Technically quantization reduces to the following two questions. Let $G$ be a reductive complex connected Lie group and $\mathfrak{g}$ its Lie algebra. Consider a semisimple conjugacy class $O$ passing through a point $t$ in a fixed maximal torus $T$ and denote by $K \subset G$ the centralizer subgroup of $t$ with the Lie algebra $\mathfrak{k} \subset \mathfrak{g}$. Let $U_{q}(\mathfrak{g})$ denote the standard quanization of the universal enveloping algebra $U(\mathfrak{g})$. Associated with $t$ is a $U_{q}(\mathfrak{g})$-module $M$ of the highest weight $\lambda=\lambda(t)$, such that the coordinate ring $\mathbb{C}[O]$ is quantized as a subalgebra $\mathbb{C}_{q}[O] \subset \operatorname{End}(M)$. Given a finite-dimensional irreducible $U_{q}(\mathfrak{g})$-module $V$, one can ask

1. what are the highest weight submodules in $V \otimes M$ ?

2. when does $V \otimes M$ split in a direct sum of those submodules?

The answer to the first question is easy for $K$ a Levi subgroup in $G$. Such are subgroups of maximal rank whose basis of simple roots is a part of the total root basis of $G$. For such a subgroup, the universal enveloping algebra is quantized as a Hopf subalgebra $U_{q}(\mathfrak{k}) \subset$ $U_{q}(\mathfrak{g})$, and the submodules of interest are parabolically induced from representations of $U_{q}(\mathfrak{k})$. Contrary to that, conjugacy classes with non-Levi $K$ have no natural quantum stabilizer in $U_{q}(\mathfrak{g})$, so description of highest weight submodules in $V \otimes M$ is not so straightforward.

The second question is challenging for both Levi and non-Levi $K$. It turns out that the answer can be formulated in terms of a contravariant form on $V \otimes M$, [M3]. The 
problem reduces to computing the determinant of a finite-dimensional matrix, which we call extremal twist. In this paper, we apply the complete reducibility criterion of [M3] to the simplest conjugacy classes with Levi stabilizer subgroups.

We consider complexification $\mathbb{P}^{n}$ of the projective space $\mathbb{C} P^{n}$ (regarded as a real affine variety). As a variety of rank-1 projectors on $\mathbb{C}^{n+1}, \mathbb{P}^{n}$ is isomorphic to a conjugacy class of the general linear group $G L(n+1)$ with the stabilizer subgroup $G L(1) \times G L(n+1)$. Specifically, different realizations of $\mathbb{P}^{n}$ by conjugacy classes with the same stabilizer give rise to different Poisson structures on $\mathbb{P}^{n}$. They are induced by the Semenov-Tian-Shanski bracket on $G L(n+1)$, [STS]. We show that almost all these Poisson structures admit quantization of vector bundles on $\mathbb{P}^{n}$. Moreover, the quantization can be specialized to a particular value of the deformation parameter $q \in \mathbb{C}$, provided it is not a root of unity and not an even root of $x_{1} x_{2}^{-1}$, where $x_{1}$ and $x_{2}$ are the two different eigenvalues of the class representative $t$.

In accordance with the Serre-Swan theorem $[\mathrm{S}, \mathrm{Sw}]$, a vector bundle $E$ over a base space $O$ translates to a sheaf of local sections equipped with the structure of a module over the sheaf of local functions on $O$. It is, in fact, a bimodule, but the two-sided action is an incidental consequence of commutativity, which is lost in quantization. So by a vector bundle over a quantum space we understand a one-sided (left or right) projective module over its noncommutative "algebra of functions". Our examples of interest are affine varieties, in the classical limit, so we simplify consideration to the setting of global functions and global sections.

Under conditions to be specified below (cf. Proposition 3.8), $V \otimes M$ splits into a direct sum of irreducible highest weight submodules parameterized by irreducible $\mathfrak{k}_{\text {-submodules in }}$ $V$. They are separated by invariant idempotents, which belong to $\operatorname{End}(V) \otimes \mathbb{C}_{q}\left[\mathbb{P}^{n}\right]$ and give rise to projective $\mathbb{C}_{q}\left[\mathbb{P}^{n}\right]$-modules identified with quantum vector bundles. In the classical limit, their fibers are the corresponding $\mathfrak{k}$-submodules in $V$.

Since $\mathbb{P}^{n}$ is a symmetric space, we take advantage of an alternative presentation of the algebra $\mathbb{C}_{q}\left[\mathbb{P}^{n}\right]$ via a quantum symmetric pair, [Let, Kolb]. There is a one-parameter family of solutions of the Reflection Equation associated with $U_{q}(\mathfrak{g})$, which define one-dimensional representations of $\mathbb{C}_{q}\left[\mathbb{P}^{n}\right]$ (regarded as "points" on the quantum projective space). Every such a solution facilitates a realization of $\mathbb{C}_{q}\left[\mathbb{P}^{n}\right]$ as a subalgebra in the Hopf dual $\mathcal{T}$ to $U_{q}(\mathfrak{g})$, [DM2]. At the same time, it defines a left coideal subalgebra $\mathcal{B} \subset U_{q}(\mathfrak{g})$ such that $\mathbb{C}_{q}\left[\mathbb{P}^{n}\right]$ is identified with the subalgebra of $\mathcal{B}$-invariants in $\mathcal{T}$ under the right translation action. The algebra $\mathcal{B}$ is a deformation of $U\left(\mathfrak{k}^{\prime}\right)$, where $\mathfrak{k}^{\prime} \simeq \mathfrak{k}$ is the isotropy Lie algebra of the "quantum 
point" in the classical limit.

We prove that every finite-dimensional $U_{q}(\mathfrak{g})$-module $V$ is completely reducible over $\mathcal{B}$ and that the simple submodules are deformations of irreducible $\mathfrak{k}^{\prime}$-submodules. They are readily constructed out of invariant idempotents from $\operatorname{End}(V) \otimes \mathbb{C}_{q}\left[\mathbb{P}^{n}\right]$ projecting $V \otimes M$ onto the corresponding irreducible submodules. This way we realize projective left $\mathbb{C}_{q}\left[\mathbb{P}^{n}\right]$ modules by $\mathcal{B}$-invariants in $\mathcal{T} \otimes X$, where $X$ is a right $\mathcal{B}$-module and $\mathcal{T}$ is equipped with the right translation action. The invariants also carry a $U_{q}(\mathfrak{g})$-action via the left translations on $\mathcal{T}$. This is a deformation of a classical realization of the equivariant vector bundle associated with a $\mathfrak{k}^{\prime}$-module $X$ (the fiber).

The set up of the paper is as follows. Section 2 contains basic facts about quantum groups and description of the base module for $\mathbb{C}_{q}\left[\mathbb{P}^{n}\right]$. At the beginning of Section 3, we summarize the results of [M3] that are relevant to the present task. We use them to study the structure of tensor products of finite-dimensional and the base module thereafter. In Section 4 , we construct quantum vector bundles as projective (right) $\mathbb{C}_{q}\left[\mathbb{P}^{n}\right]$-modules, based on results of Section 3. Then we switch to quantum symmetric pairs and give a realization of projective (left) $\mathbb{C}_{q}\left[\mathbb{P}^{n}\right]$-modules through $\mathcal{B}$-invariants, similarly to the classical induced representations.

\section{Preliminaries}

Denote by $\mathfrak{g}$ the general linear algebra of complex $(n+1) \times(n+1)$-matrices. Fix the Cartan subalgebra $\mathfrak{h} \subset \mathfrak{g}$ as the span of diagonal matrices and identify it with its dual vector space $\mathfrak{h}^{*}$ via the trace inner product $(.,$.$) . The root system \mathrm{R}$ of $\mathfrak{g}$ is expressed through the standard orthornomal basis $\left\{\varepsilon_{i}\right\}_{i=1}^{n} \subset \mathfrak{h}^{*}$ as $\left\{\varepsilon_{i}-\varepsilon_{j}\right\}_{i \neq j}$ with the basis of simple positive roots $\Pi^{+}=\left\{\alpha_{i}\right\}_{i=1}^{n}$, where $\alpha_{i}=\varepsilon_{i}-\varepsilon_{i+1}, i=1, \ldots, n$. The subset $\mathrm{R}^{+}$of all positive roots is $\left\{\varepsilon_{i}-\varepsilon_{j}\right\}_{i<j}$.

For all $\lambda \in \mathfrak{h}^{*}$ denote by $h_{\lambda}$ the element of $\mathfrak{h}$ satisfying $\mu\left(h_{\lambda}\right)=(\mu, \lambda)$, for all $\mu \in \mathfrak{h}^{*}$. Denote by $\rho \in \mathfrak{h}^{*}$ the half-sum of positive roots.

Suppose that $q \in \mathbb{C}$ is invertible and not a root of unity. In what follows, we use the shortcuts $\bar{q}=q^{-1}$ and $[x, y]_{a}=x y-a y x$ for $a \in \mathbb{C}$.

By $U_{q}(\mathfrak{g})$ we understand the standard quantum group [Dr, ChP] generated over the complex field by $e_{\alpha}, f_{\alpha}$, and $q^{ \pm h_{\alpha}}, \alpha \in \Pi^{+}$, subject to

$$
q^{ \pm h_{\alpha}} e_{\beta}=q^{ \pm(\alpha, \beta)} e_{\beta} q^{ \pm h_{\alpha}}, \quad\left[e_{\alpha}, f_{\beta}\right]=\delta_{\alpha, \beta}\left[h_{\alpha}\right]_{q}, \quad q^{ \pm h_{\alpha}} f_{\beta}=q^{\mp(\alpha, \beta)} f_{\beta} q^{ \pm h_{\alpha}}, \quad \alpha \in \Pi^{+}
$$


where $\left[h_{\alpha}\right]_{q}=\frac{q^{h_{\alpha}-q^{-h}}}{q-q^{-1}}$, and $q^{h_{\alpha}} q^{-h_{\alpha}}=1=q^{-h_{\alpha}} q^{h_{\alpha}}$. The generators $e_{\alpha}$ and $e_{-\alpha}=f_{\alpha}$ satisfy the q-Serre relations

$$
\left[e_{ \pm \alpha},\left[e_{ \pm \alpha}, e_{ \pm \beta}\right]_{q}\right]_{\bar{q}}=0, \quad \text { if } \quad(\alpha, \beta)=-1, \quad \text { and } \quad\left[e_{ \pm \alpha}, e_{ \pm \beta}\right]=0 \quad \text { if } \quad(\alpha, \beta)=0
$$

Fix the comultiplication on $U_{q}(\mathfrak{g})$ as

$$
\Delta\left(f_{\alpha}\right)=f_{\alpha} \otimes 1+q^{-h_{\alpha}} \otimes f_{\alpha}, \quad \Delta\left(q^{ \pm h_{\alpha}}\right)=q^{ \pm h_{\alpha}} \otimes q^{ \pm h_{\alpha}}, \quad \Delta\left(e_{\alpha}\right)=e_{\alpha} \otimes q^{h_{\alpha}}+1 \otimes e_{\alpha} .
$$

Then the antipode acts on the generators by the assignment

$$
\gamma\left(f_{\alpha}\right)=-q^{h_{\alpha}} f_{\alpha}, \quad \gamma\left(q^{ \pm h_{\alpha}}\right)=q^{\mp h_{\alpha}}, \quad \gamma\left(e_{\alpha}\right)=-e_{\alpha} q^{-h_{\alpha}}
$$

The counit homomorphism $\epsilon: U_{q}(\mathfrak{g}) \rightarrow \mathbb{C}$ returns on the generators $\epsilon\left(e_{\alpha}\right)=\epsilon\left(f_{\alpha}\right)=0$, and $\epsilon\left(q^{ \pm h_{\alpha}}\right)=1$.

The assignment

$$
\sigma: e_{\alpha} \rightarrow f_{\alpha}, \quad \sigma: f_{\alpha} \rightarrow e_{\alpha}, \quad \sigma: h_{\alpha} \rightarrow-h_{\alpha}
$$

extends to an algebra involutive automorphism of $U_{q}(\mathfrak{g})$. The involution $\omega=\gamma^{-1} \circ \sigma$ preserves comultiplication and is an algebra anti-automorphism of $U_{q}(\mathfrak{g})$.

Denote by $U_{q}(\mathfrak{h}), U_{q}\left(\mathfrak{g}_{+}\right), U_{q}\left(\mathfrak{g}_{-}\right)$the subalgebras in $U_{q}(\mathfrak{g})$ generated by $\left\{q^{ \pm h_{\alpha}}\right\}_{\alpha \in \Pi^{+}}$, $\left\{e_{\alpha}\right\}_{\alpha \in \Pi^{+}}$, and $\left\{f_{\alpha}\right\}_{\alpha \in \Pi^{+}}$, respectively. The quantum Borel subgroups $U_{q}\left(\mathfrak{b}_{ \pm}\right)=U_{q}\left(\mathfrak{g}_{ \pm}\right) U_{q}(\mathfrak{h})$ are Hopf subalgebras in $U_{q}(\mathfrak{g})$. Let $\mathfrak{k}$ denote the subalgebra $\mathfrak{g l}(1) \oplus \mathfrak{g l}(n) \subset \mathfrak{g}$ with the basis of simple roots $\Pi_{\mathfrak{k}}^{+}=\left\{\alpha_{2}, \ldots, \alpha_{n}\right\}$. The elements $\left\{e_{\alpha}, f_{\alpha}, q^{ \pm h_{\alpha}}\right\}_{\alpha \in \Pi_{\mathfrak{k}}^{+}}$generate a quantum subgroup $U_{q}(\mathfrak{k}) \subset U_{q}(\mathfrak{g})$. A Hopf subalgebra $U_{q}(\mathfrak{p})=U_{q}(\mathfrak{k}) U_{q}\left(\mathfrak{b}_{+}\right)$is a quantization of $U(\mathfrak{p})$, where $\mathfrak{p}=\mathfrak{k}+\mathfrak{g}_{-}$is a parabolic Lie subalgebra.

All $U_{q}(\mathfrak{g})$-modules under consideration are $U_{q}(\mathfrak{h})$-diagonalized. For any $U_{q}(\mathfrak{h})$-module $V$ we denote by $V[\mu] \subset V$ the subspace of weight $\mu \in \mathfrak{h}^{*}$, i. e., the set of vectors $v \in V$ satisfying $q^{h_{\alpha}} v=q^{(\mu, \alpha)} v$ for all $\alpha \in \Pi^{+}$. The set of weights of $V$ is denoted by $\Lambda(V)$.

\section{Base module for quantum projective space}

\subsection{Description of base module}

The complex projective space $\mathbb{C} P^{n}$ a real affine variety and can be realized as a set of Hermitian projectors in $\operatorname{End}\left(\mathbb{C}^{n+1}\right)$ of complex rank 1 . It is a real form of the complex variety of projectors on $\mathbb{C}^{n+1}$ with unit trace. The latter is a homogeneous $G L(n+1)$-variety that is isomorphic to a conjugacy class passing through a matrix $t=\operatorname{diag}\left(x_{1}, \ldots, x_{n}\right) \in G L(n+1)$ 
with $x_{i}=x_{j}$ for $i, j \geqslant 2$. The centralizer of $t$ is the subgroup $G L(1) \times G L(n)$ whose Lie algebra is $\mathfrak{k}$.

By quantization of the affine ring $\mathbb{C}\left[\mathbb{P}^{n}\right]$ we call a $\mathbb{C}\left[q, q^{-1}\right]$-algebra $\mathbb{C}_{q}\left[\mathbb{P}^{n}\right]$, which is a free module when extended over the local ring of rational functions in $q$ regular at $q=1$ and which is isomorphic to $\mathbb{C}\left[\mathbb{P}^{n}\right]$ in the classical limit $q \rightarrow 1$. We assume that $\mathbb{C}_{q}\left[\mathbb{P}^{n}\right]$ is a $U_{q}(\mathfrak{g})$ module algebra, which structure is a deformation of the classical structure of $U(\mathfrak{g})$-module algebra on $\mathbb{C}\left[\mathbb{P}^{n}\right]$.

The quantization $\mathbb{C}_{q}\left[\mathbb{P}^{n}\right]$ does exist and can be realized as a $U_{q}(\mathfrak{g})$-invariant subalgebra of of linear operators on a highest weight module $M$, which we call base module for $\mathbb{P}^{n}$, [M1]. Base modules form a 2-parameter family delivering different quantizations of $\mathbb{C}\left[\mathbb{P}^{n}\right]$. They are in bijection with diagonal matrices whose centralizer Lie algebra is $\mathfrak{k}$.

The module $M$ has a PBW basis that makes it isomorphic to the vector space of polynomials in $n$ variables. To describe this basis, let us introduce compound root vectors, cf. $[\mathrm{ChP}]$. For $\alpha=\alpha_{i}+\ldots+\alpha_{j} \in \mathrm{R}^{+}$with $i<j$ put

$$
e_{\alpha}=\left[e_{\alpha_{j}}, \ldots\left[e_{\alpha_{i+1}}, e_{\alpha_{i}}\right]_{q} \ldots\right]_{q}, \quad f_{\alpha}=\left[\ldots\left[f_{\alpha_{i}}, f_{\alpha_{i+1}}\right]_{\bar{q}}, \ldots f_{\alpha_{j}}\right]_{\bar{q}} .
$$

We reserve the notation $\beta_{i}$ for the weights $\beta_{i}=\alpha_{1}+\ldots+\alpha_{i}, i=1, \ldots, n$.

Let $\lambda$ be a weight such that $q^{\left(\lambda, \varepsilon_{i}\right)}=\sqrt{x_{i}}$ assuming the same root value for $i>1$. It defines a 1-dimensional representation of the parabolic subalgebra $U_{q}(\mathfrak{p})$ which is zero on $e_{\alpha}$ and $f_{\alpha}$ and returns $q^{\left(\lambda, \varepsilon_{i}\right)}$ on all $q^{h_{\varepsilon_{i}}}$. Consider the parabolic $U_{q}(\mathfrak{p})$-module $M$ of highest weight $\lambda$ induced by the character of $U_{q}(\mathfrak{p})$ with canonical generator $1_{\lambda} \in M$. It is a quotient of the ordinary Verma module $\hat{M}_{\lambda}$ by the sum of submodules generated by $f_{\alpha} 1_{\lambda}, \alpha \in \Pi_{\mathfrak{k}}^{+}$. The module $M$ has a basis of vectors $f_{\beta_{1}}^{m_{1}} \ldots f_{\beta_{n}}^{m_{n}} 1_{\lambda}$, where $m_{i}$ take all possible values in $\mathbb{Z}_{+}$.

Pick up $s \in \mathbb{C}$ such that $q^{s}=q^{\left(\lambda, \alpha_{1}\right)}$ and put $|\vec{m}|_{i}=m_{i}+\ldots+m_{n}$ for all $i=1, \ldots, n$. Clearly $q^{2 s}=x_{1} x_{2}^{-1}$.

Lemma 3.1. The action of $U_{q}(\mathfrak{g})$ on $M$ is given by

$$
\begin{aligned}
e_{\alpha_{1}} f_{\beta_{1}}^{m_{1}} \ldots f_{\beta_{n}}^{m_{n}} 1_{\lambda} & =\left[m_{1}\right]_{q}\left[s-|\vec{m}|_{1}+1\right]_{q} f_{\beta_{1}}^{m_{1}-1} f_{\beta_{2}}^{m_{2}} \ldots f_{\beta_{n}}^{m_{n}} 1_{\lambda}, \\
e_{\alpha_{i}} f_{\beta_{1}}^{m_{1}} \ldots f_{\beta_{n}}^{m_{n}} 1_{\lambda}, & =-\left[m_{i}\right]_{q} q^{-m_{i}-1-s} f_{\beta_{1}}^{m_{1}} \ldots f_{\beta_{i-1}}^{m_{i-1}+1} f_{\beta_{i}}^{m_{i}-1} \ldots f_{\beta_{n}}^{m_{n}} 1_{\lambda}, \quad i>1, \\
f_{\alpha_{1}} f_{\beta_{1}}^{m_{1}} \ldots f_{\beta_{n}}^{m_{n}} 1_{\lambda} & =f_{\beta_{1}}^{m_{1}+1} f_{\beta_{2}}^{m_{2}} \ldots f_{\beta_{n}}^{m_{n}} 1_{\lambda}, \\
f_{\alpha_{i}} f_{\beta_{1}}^{m_{1}} \ldots f_{\beta_{n}}^{m_{n}} 1_{\lambda} & =-q^{-1}\left[m_{i-1}\right]_{q} f_{\beta_{1}}^{m_{1}} \ldots f_{\beta_{i-1}}^{m_{i-1}-1} f_{\beta_{i}}^{m_{i}+1} \ldots f_{\beta_{n}}^{m_{n}} 1_{\lambda}, \quad i>1 .
\end{aligned}
$$

Proof. Direct caculation. 
Recall that a weight vector in a $U_{q}(\mathfrak{g})$-module is called singular if it is killed by all $e_{\alpha}, \alpha \in \Pi^{+}$. Singular vectors generate submodules of highest weight.

Corollary 3.2. The module $M$ is irreducible if and only if $[s-m]_{q} \neq 0$ for all $m \in \mathbb{Z}_{+}$.

Proof. A singular vector in $M$ appears only when $q^{2 s} \in q^{2 \mathbb{N}}$ and it is proportional to $f_{\beta_{1}}^{m} 1_{\lambda}$.

\subsection{Canonical contravariant form on $V \otimes M$ and extremal twist}

In this section we apply a criterion for a tensor product $V \otimes M$ to be completely reducible, following [M3]. Recall that a bilinear form $\langle.,$.$\rangle on a module V$ is called contravariant with respect to involution $\omega$ if for all $v, w \in V$ and all $h \in U_{q}(\mathfrak{g})$ it satisfies $\langle h v, w\rangle=\langle v, \omega(h) w\rangle$. It is known that every highest weight module has a unique, up to a scalar multiplier, contravariant form, which is non-degenerate if and only if the module is irreducible. It is called Shapovalov form of the highest weight module.

Suppose that $V$ is an irreducible finite-dimensional $U_{q}(\mathfrak{g})$-module. Denote by $(V \otimes M)^{+}$ the subspace of singular vectors in $V \otimes M$. Introduce a canonical contravariant bilinear form on $V \otimes M$ as the product of Shapovalov forms on $V$ and $M$. Since $V$ and $M$ are irreducible, the canonical form is non-degenerate on $V \otimes Z$.

Theorem 3.3 ([M3]). The tensor product $V \otimes M$ is completely reducible if and only if the canonical form is non-degenerate when restricted to $(V \otimes M)^{+}$.

Next we describe a method of computing the determinant of the canonical form on $(V \otimes M)^{+}$that we use in what follows. Let $\nu$ denote the highest (positive dominant) weight of $V$ and put $\ell_{i}=\left(\nu+\rho, \alpha_{i}\right)-1 \in \mathbb{Z}_{+}, i=1, \ldots, n$. There are two natural parameterizations of $(V \otimes M)^{+}$with subspaces in $V$ and $M$. One of them is by the span $V_{M}^{+}$of $U_{q}(\mathfrak{k})$ singular vectors, i. e., those annihilated by $e_{\alpha}, \alpha \in \Pi_{\mathfrak{k}}^{+}$(Gelfant-Zeitlin reduction). Then $\Lambda\left(V_{M}^{+}\right)=\left\{\nu-\sum_{i=1}^{n} m_{i} \beta_{i}\right\}$ with $0 \leqslant m_{i} \leqslant \ell_{i}$ for all $i$, and all weights are multiplicity-free.

The alternative parametrization of $(V \otimes M)^{+}$is by a vector subspace $M_{V}^{+} \subset M$. Define a left ideal $I_{V}^{+} \subset U_{q}\left(\mathfrak{g}_{+}\right)$generated by $\left\{e_{\alpha_{i}}^{\ell_{i}+1}\right\}_{i=1}^{n}$. Set $M_{V}^{+} \subset M$ as the nil-space of $I_{V}^{+}$. The linear isomorphism between $M_{V}^{+}$and $(V \otimes M)^{+}$acts by the assignment $(V \otimes M)^{+} \ni u=$ $\sum_{i} v_{i} \otimes w_{i} \mapsto \sum_{i}\left\langle v_{i}, 1_{\nu}\right\rangle w_{i} \in M_{V}^{+}$, where $1_{\nu}$ is the highest vector in $V$.

Lemma 3.4. The module $M$ splits into the orthogonal sum $M=M_{V}^{+} \oplus \omega\left(I_{V}^{+}\right) M$ with

$$
M_{V}^{+}=\operatorname{Span}\left\{f_{\beta_{1}}^{m_{1}} \ldots f_{\beta_{n}}^{m_{n}} 1_{\lambda}\right\}_{m_{1} \leqslant \ell_{1}, \ldots, m_{n} \leqslant \ell_{n}}, \quad \omega\left(I_{V}^{+}\right) M=\operatorname{Span}\left\{f_{\beta_{1}}^{k_{1}} \ldots f_{\beta_{n}}^{k_{n}} 1_{\lambda}\right\}_{k_{1}, \ldots, k_{n}},
$$

where $k_{i}>\ell_{i}$ for some $i=1, \ldots, n$. 
Proof. Observe that $\omega\left(I_{V}^{+}\right) M=\sum_{i=1}^{n} f_{\alpha_{i}}^{\ell_{i}+1} M$, which proves the right equality. The left equality follows from Lemma 3.1. This implies the decomposition of $M$.

Introduce a linear operator $\theta_{M, Z} \in \operatorname{End}\left(M_{Z}^{+}\right)$as follows. Pick up a singular vector $u \in M \otimes V \simeq V \otimes M$ and present it as $u=\sum_{i} w_{i} \otimes f_{i} 1_{\nu}=w \otimes 1_{\nu}+\ldots$ Put $\theta_{M, V}(w)$ equal to the image of $\sum_{i} \gamma^{-1}\left(f_{i}\right) w_{i} \in M$ under the orthogonal projection to $M_{V}^{+}$. This operator is independent of the chosen presentation of $u$. We can regard $\theta_{M, V}$ as a bilinear form $\left\langle\theta_{V, Z}(),.\right\rangle: M_{V}^{+} \otimes M_{V}^{+} \rightarrow \mathbb{C}$.

Proposition $3.5([\mathrm{M} 3])$. The form $\left\langle\theta_{M, V}(),.\right\rangle$ is the pullback of the canonical form restrected to $(M \otimes V)^{+}$, under the isomorphism $M_{V}^{+} \rightarrow(M \otimes V)^{+}$.

Under certain conditions, see [M3], Proposition 7.1, the operator $\theta_{M, V}$ can be obtained from a lift $\mathcal{F} \in U_{q}\left(\mathfrak{g}_{+}\right) \hat{\otimes} U_{q}\left(\mathfrak{g}_{-}\right)$of the inverse invariant pairing between an irreducible Verma module $\hat{M}_{\mu}$ (of highest weight $\mu$ ) and its right dual ${ }^{*} \hat{M}_{\mu}$ (the opposite Verma module of lowest weight $-\mu$ ). Such a lift is the image of $1 \in \mathbb{C}$ under the composition of maps

$$
\mathbb{C} \rightarrow{ }^{*} \hat{M}_{\mu} \hat{\otimes} \hat{M}_{\mu} \rightarrow U_{q}\left(\mathfrak{g}_{+}\right) \hat{\otimes} U_{q}\left(\mathfrak{g}_{-}\right) \quad \text { (a completed tensor products) },
$$

where the right arrow is a $\mathbb{C}$-linear section of the natural homomorphism of $U_{q}\left(\mathfrak{g}_{+}\right) \otimes U_{q}\left(\mathfrak{g}_{-}\right)$modules (the left arrow is the coevaluation map). Using the Sweedler notation $\mathcal{F}_{1} \otimes \mathcal{F}_{2}$ for $\mathcal{F}$ we set $\Upsilon_{\mu}=\gamma^{-1}\left(\mathcal{F}_{2}\right) \mathcal{F}_{1} \in U_{q}(\mathfrak{g})$ (a certain extension of the quantum group assumed). The module $V$ is a quotient of $\hat{M}_{\nu}$, therefore the lift $\mathcal{F}$ has poles at $\mu=\nu$. However, its part that is relevant to $M_{V}^{+}$is well-defined if the following hypothesis holds true.

Proposition 3.6 ([M3]). Suppose that every singular vector in $V \otimes M$ is the image of a singular vector in $\hat{M}_{\mu} \otimes M$ under the natural homomorphism $\hat{M}_{\mu} \otimes M \rightarrow V \otimes M$. Then $\left\langle\theta_{M, V}(),.\right\rangle=\lim _{\mu \rightarrow \nu}\left\langle\Upsilon_{\mu}(),.\right\rangle$ on $M_{V}^{+}$.

\subsection{Complete reduciblility of $V \otimes M$}

The inverse of the operator $\Upsilon_{\mu}$ is found in [EV], in connection with the dynamical Weyl group. Their result can be used for calculation of $\theta_{M, V}$ because the conditions of Proposition 3.6 are fulfilled, as proved below.

Proposition 3.7. All singular vectors in $V \otimes M$ are images of singular vectors in $\hat{M}_{\nu} \otimes M$.

Proof. Thanks to the presence of the Gelfand-Zeitlin basis in $V$ (see e. g., [Mol] for the classical group version), every element of $v \in V_{M}^{+}$can be presented as $v=f(\nu) 1_{\nu}$, where 
$f(\nu) \in U_{q}\left(\mathfrak{g}_{-}\right)$belongs to the normalizer of the left ideal $U_{q}(\mathfrak{g}) I_{M}^{+}$in $U_{q}(\mathfrak{g})$ (the quotient of the normalizer by $U_{q}(\mathfrak{g}) I_{M}^{+}$is the quantum Mickelsson algebra of the pair $(\mathfrak{g}, \mathfrak{k})$, cf. $\left.[\mathrm{KO}]\right)$. Therefore $v$ is the image of an element $\hat{v} \in\left(\hat{M}_{\nu}\right)_{M}^{+} \simeq\left(\hat{M}_{\nu} \otimes M\right)^{+}$. The corresponding singular vectors in $\hat{M}_{\nu} \otimes M$ and $V \otimes M$ are, respectively, $\hat{u}=\mathcal{F}_{M}\left(\hat{v} \otimes 1_{\lambda}\right)$ and $u=\mathcal{F}_{M}\left(v \otimes 1_{\lambda}\right)$, where $\mathcal{F}_{M} \in U_{q}\left(\mathfrak{g}_{+}\right) \hat{\otimes} U_{q}\left(\mathfrak{g}_{-}\right)$is a lift of the inverse invariant pairing between $M$ and ${ }^{*} M$. Then $u$ is the image of $\hat{u}$.

Since the weights in $M$ are multiplicity free, we can write, following [EV],

$$
\Upsilon_{\nu}(w) \propto \prod_{\alpha \in \mathrm{R}_{\mathfrak{g}}^{+}} \prod_{k=1}^{l_{\xi, \alpha}} \frac{[(\nu+\rho+\xi, \alpha)+k]_{q}}{[(\nu+\rho, \alpha)-k]_{q}} w, \quad w \in M_{V}^{+}[\xi],
$$

where $l_{\xi, \alpha}=\max \left\{l \in \mathbb{Z}: e_{\alpha}^{l} w \neq 0\right\}$. The operator $\Upsilon_{\nu}$ preserves $M_{V}^{+}$, thus $\theta_{M, V}=\Upsilon_{\nu}$ on $M_{V}^{+}$.

Proposition 3.8. The operators $\theta_{M, V}$ are invertible for all $V$ iff $q^{2 s} \notin q^{2 \mathbb{Z}}$.

Proof. Let $\xi$ be a weight from $\Lambda\left(M_{V}^{+}\right)$, that is, $\xi=\lambda-\sum_{i=1}^{n} m_{i} \beta_{i}$ with $0 \leqslant m_{i} \leqslant \ell_{i}=$ $\left(\nu+\rho, \alpha_{i}\right)-1$. It is easy to check, using Lemma 3.1, that for $\alpha=\varepsilon_{i}-\varepsilon_{j+1}, i \leqslant j+1$, the integer $l_{\xi, \alpha}$ equals $m_{j}$. Then the factor $[(\nu+\rho, \alpha)-k]_{q}$ equals $\left[m_{i}+\ldots+m_{j}+(j-i+1)-k\right]_{q} \neq 0$, as $q$ is not a root of unity. Therefore the denominator of $\theta_{M, V}$ does not vanish.

Define $\phi_{\xi, \alpha, k}=[(\nu+\rho+\xi, \alpha)+k]_{q}$ for all $\xi \in \Lambda\left(M_{V}^{+}\right), \alpha \in \mathrm{R}^{+}$, and $k \in \mathbb{N}$. Then $\operatorname{det} \theta_{M, V}$ is equal to the product $\prod_{\xi \in \Lambda\left(M_{V}^{+}\right)} \prod_{\alpha \in \mathrm{R}_{\mathfrak{g}}^{+}} \prod_{k=1}^{l_{\xi, \alpha}} \phi_{\xi, \alpha, k}$, up to a non-zero factor.

If $\alpha \in \Pi_{\mathfrak{k}}^{+}$, then $(\nu+\rho+\xi, \alpha)+k \in \mathbb{Z} \backslash\{0\}$, and $\phi_{\xi, \alpha, k} \neq 0$ since $q$ is not a root of unity. Suppose that $\alpha=\beta_{j}$. Then $\phi_{\xi, \beta_{j}, k}=\left[\left(\nu+\rho, \beta_{j}\right)-\sum_{i=1}^{n} m_{i}\left(\beta_{i}, \beta_{j}\right)+k+s\right]_{q}$. The first three terms are obviously integer, therefore $\operatorname{det} \theta_{M, V} \neq 0$ if $s$ satisfies the hypothesis.

Conversely, suppose that $[s-m]_{q}=0$ for some $m \in \mathbb{Z}$ and show that $\operatorname{det} \theta_{M, V}=0$ if $\ell_{1}$ is sufficiently large. Put $m_{i}=0$ for $i>1$, then $1+\ell_{1}-2 m_{1}+k$ with $m_{1} \in\left[0, \ell_{1}\right]$ and $k \in\left[1, m_{1}\right]$ takes all integer values in the interval $\left[2-\ell_{1}, \ell_{1}\right]$. Therefore, one of the factors $\phi_{\xi, \beta_{1}, k}$ vanishes once $|m-1| \leqslant \ell_{1}-1$.

Corollary 3.9. Under the assumptions of Proposition 3.8, the modules $V \otimes M$ are completely reducible for all finite-dimensional $V$. 


\section{Equivariant vector bundles over quantum projective spaces}

\subsection{Parabolic Verma modules}

The base module $M$ is an example of parabolic induction from a one-dimensional representation of $U_{q}(\mathfrak{k})$. Let us remind this construction in general. The Lie subalgebras $\mathfrak{p}_{ \pm}=\mathfrak{k}+\mathfrak{g}_{ \pm} \subset \mathfrak{g}$ are called parabolic with the Levi factor $\mathfrak{k}$. Their nilradicals $\mathfrak{n}_{ \pm}$are spanned by the vectors of roots from $R_{\mathfrak{g}}^{ \pm} \backslash R_{\mathfrak{k}}^{ \pm}=\left\{ \pm \beta_{i}\right\}_{i=1}^{n}$ respectively, thus $\mathfrak{p}_{ \pm}=\mathfrak{k} \ltimes \mathfrak{n}_{ \pm}$. Denote by $U_{q}\left(\mathfrak{n}_{ \pm}\right)$the subalgebra in $U_{q}(\mathfrak{g})$ generated by $\left\{e_{\beta_{i}}\right\}_{i=1}^{n}$, and similarly by $U_{q}\left(\mathfrak{n}_{-}\right)$the subalgebra generated by $\left\{f_{\beta_{i}}\right\}_{i=1}^{n}$. The algebra $U_{q}(\mathfrak{g})$ enjoys a factorization $U_{q}(\mathfrak{g})=U_{q}\left(\mathfrak{n}_{\mp}\right) U_{q}\left(\mathfrak{p}_{ \pm}\right)$with $U_{q}\left(\mathfrak{p}_{ \pm}\right)=U_{q}(\mathfrak{k}) U_{q}\left(\mathfrak{n}_{ \pm}\right)$, facilitated by the PBW basis.

Fix a finite-dimensional irreducible $U_{q}(\mathfrak{k})$-module $X$ of highest weight $\xi$ and make it a module over $U_{q}\left(\mathfrak{p}_{+}\right)$assuming the trivial action of $U_{q}\left(\mathfrak{n}_{+}\right)$. Denote by $M_{X}$ the induced module $U_{q}(\mathfrak{g}) \otimes_{U_{q}\left(\mathfrak{p}_{+}\right)} X$. One similarly defines parabolic Verma modules of lowest weight, $N_{X}=U_{q}(\mathfrak{g}) \otimes_{U_{q}\left(\mathfrak{p}_{-}\right)} X$. Applying Frobenius reciprocity twice, one can check that $M_{X} \otimes N_{Y}$ is isomorphic to the $U_{q}(\mathfrak{g})$-module induced from the $U_{q}(\mathfrak{k})$-module $X \otimes Y$.

Recall that the character $\operatorname{ch}(W)$ of a $U_{q}(\mathfrak{h})$-module $W$ is defined as the formal sum $\sum_{\mu \in \Lambda(W)} \operatorname{dim} W[\mu] q^{\mu}$, where $q^{\mu}$ is a homomorphism $U_{q}(\mathfrak{h}) \rightarrow \mathbb{C}$ acting by $q^{h_{\alpha}}=q^{(\mu, \alpha)}$ for all $\alpha \in \Pi^{+}$. For a pair of $U_{q}(\mathfrak{h})$-modules we write $\operatorname{ch}\left(W_{1}\right) \leqslant \operatorname{ch}\left(W_{2}\right)$ if $\operatorname{dim} W_{1}[\mu] \leqslant \operatorname{dim} W_{2}[\mu]$ for all $\mu$. The triangular factorization of $U_{q}(\mathfrak{g})$ implies that $M_{X} \simeq U_{q}\left(\mathfrak{n}_{-}\right) X$. Therefore the character of $M_{X}$ is equal to $\operatorname{ch}\left(M_{\mathbb{C}}\right)(\operatorname{ch} X)$, where $M_{\mathbb{C}}$ is parabolically induced from the trivial $U_{q}(\mathfrak{k})$-module.

Given a weight $\lambda$ subject to $q^{2(\lambda, \alpha)}=1$ for all $\alpha \in \Pi_{\mathfrak{k}}^{+}$, one can define a one-dimensional $U_{q}(\mathfrak{k})$-module $\mathbb{C}_{\lambda}$ where $U_{q}(\mathfrak{h})$ acts by $q^{h_{\alpha}} \mapsto q^{(\lambda, \alpha)}$. Then for any finite-dimensional $U_{q}(\mathfrak{k})$ module $X$ we set $X_{\lambda}=X \otimes \mathbb{C}_{\lambda}$. We fix $\lambda$ to be the highest weight of the base module $M$.

Theorem 4.1. Let $V$ be a finite-dimensional $U_{q}(\mathfrak{g})$-module and $V=\oplus_{i} X^{i}$ its irreducible decomposition over $U_{q}(\mathfrak{k})$. Suppose that $q^{2\left(\lambda, \alpha_{1}\right)} \notin q^{2 \mathbb{Z}}$. Then $V \otimes M \simeq \oplus_{i} M_{X_{\lambda}^{i}}$ is an irreducible decomposition.

Proof. Since $M$ is irreducible, $\operatorname{Hom}\left(M_{X_{\lambda}^{i}}, V \otimes M\right) \simeq \operatorname{Hom}\left(M_{X_{\lambda}^{i}} \otimes N, V\right) \simeq \operatorname{Hom}_{U_{q}(\mathfrak{k})}\left(X^{i}, V\right)$. We will prove that all homomorphisms $M_{X_{\lambda}^{i}} \rightarrow V \otimes M$ are embeddings. Let $M_{i}$ denote the image of $M_{X_{\lambda}^{i}}$. By Proposition 3.8, $V \otimes M=\oplus_{i} M_{i}$ is an irreducible decomposition. Therefore $\sum_{i} \operatorname{ch}\left(M_{i}\right)=\operatorname{ch}(M) \operatorname{ch}(V)$. On the other hand, $\sum_{i} \operatorname{ch}\left(M_{X_{\lambda}^{i}}\right)=\sum_{i} \operatorname{ch}(M) \operatorname{ch}\left(X^{i}\right)=$ 
$\operatorname{ch}(M) \operatorname{ch}(V)$. Since $\operatorname{ch}\left(M_{i}\right) \leqslant \operatorname{ch}\left(M_{X_{\lambda}^{i}}\right)$, that is possible only if $\operatorname{ch}\left(M_{i}\right)=\operatorname{ch}\left(M_{X_{\lambda}^{i}}\right)$ and therefore $M_{i} \simeq M_{X_{\lambda}^{i}}$ for all $i$.

Note that one cannot relax the condition on the weight $\lambda$ as $V$ varies over all finitedimensional modules.

Remark that $x_{i}=q^{2\left(\lambda, \varepsilon_{i}\right)}$ should be treated as constants rather than functions of $q$ (e.g., via rescaling of $\lambda$ ). They have the meaning of eigenvalues of matrices comprising the conjugacy class that represents $\mathbb{P}^{n}$. Once $x_{1} x_{2}^{-1} \notin q^{2 \mathbb{Z}}$, the modules $M_{X_{\lambda}}$ can be extended over the ring of formal power series $\mathbb{C}[[\hbar]]$ via $q=e^{\hbar}$. This trick will be used in the next section in the quantization context.

\subsection{Projective modules over $\mathbb{C}_{q}\left[\mathbb{P}^{n}\right]$}

By a classical equivariant vector bundle over $\mathbb{P}^{n}$ with fiber $X$ we understand the (left or right) projective $\mathbb{C}\left[\mathbb{P}^{n}\right]$-module of global sections denoted by $\Gamma\left(\mathbb{P}^{n}, X\right)$. It can be realized as the subspace of $\mathfrak{k}$-invariants in $\mathbb{C}[G] \otimes X, G=G L(n+1)$, where $\mathfrak{k}$ acts on $\mathbb{C}[G]$ by translations.

We understand quantization as deformation, when complex vector spaces are extended to free $\mathbb{C}[[\hbar]]$-modules. In our situation, when all modules and maps are rational in $q$, they can be considered over the local ring of rational functions in $q$ regular at $q=1$. They can be specialized at generic $q$ meaning that $q$ can can take all but a finite set of complex numbers including $q=1$, in every submodule of finite rank.

Let $\mathcal{A} \subset \operatorname{End}(M)$ denote the quantized polynomial algebra $\mathbb{C}_{q}\left[\mathbb{P}^{n}\right]$. By quantization of an equivariant vector bundle on $\mathbb{P}^{n}$ in this subsection we mean a $U_{q}(\mathfrak{g})$-equivariant deformation of $\Gamma\left(\mathbb{P}^{n}, X\right)$ in the class of right $\mathbb{C}_{q}\left[\mathbb{P}^{n}\right]$-modules. We will realize it as $\hat{P}(V \otimes \mathcal{A})$, where $V \supset X$ is a finite-dimensional $U_{q}(\mathfrak{g})$-module and $\hat{P} \in \operatorname{End}(V) \otimes \mathcal{A}$ is an $U_{q}(\mathfrak{g})$-invariant idempotent. Such idempotents can be constructed via a direct sum decomposition of $V \otimes M$ due to the following fact.

Proposition 4.2. For all $q$ except for a finite set, every invariant projector from $V \otimes M$ onto an irreducible submodule belongs to $\operatorname{End}(V) \otimes \mathcal{A}$.

Proof. For generic $q$, the algebra $\mathcal{A}$ is embedded in the locally finite part $\operatorname{End}^{\circ}(M)$ of the adjoint $U_{q}(\mathfrak{g})$-module $\operatorname{End}(M)$, which is isomorphic to the locally finite part of $M \otimes N$. Frobenius reciprocity yields

$$
\operatorname{Hom}_{U_{q}(\mathfrak{g})}(M \otimes N, V) \simeq \operatorname{Hom}_{U_{q}(\mathfrak{g})}(M, V \otimes M) \simeq \operatorname{Hom}_{\mathfrak{k}}(\mathbb{C}, V) \simeq \operatorname{Hom}_{U_{q}(\mathfrak{g})}(\mathcal{A}, V)
$$


for every finite-dimensional $U_{q}(\mathfrak{g})$-module $V$. Therefore the image of $\mathcal{A}$ in $\operatorname{End}(M)$ coincides with $\operatorname{End}^{\circ}(M)$ (this is a quantum group version of Kostant's problem, cf. [KST]). An invariant projector $\hat{P} \in \operatorname{End}(V \otimes M)$ is a matrix with entries in $\operatorname{End}^{\circ}(M)$, therefore it is in $\operatorname{End}(V) \otimes \mathcal{A}$. Since $\hat{P}$ is rational in $q$ and all isotypic components of $\mathcal{A}$ are finite-dimensional, this is true for all but a finite number of values of $q$.

Irreducible decomposition of $V \otimes M$ depends on a choice of basis in $V^{\mathfrak{k}_{+}}=V_{M}^{+}$. Let $\hat{P} \in \operatorname{End}(V) \otimes \mathcal{A}$ be the projector to a particular copy of $M_{X_{\lambda}}$ determined by $v \in V^{\mathfrak{k}_{+}} \cap X$.

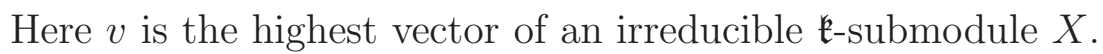

Theorem 4.3. The right $\mathcal{A}$-module $\hat{P}(V \otimes \mathcal{A})$ is a quantization of $\Gamma\left(\mathbb{P}^{n}, X\right)$.

Proof. The left $U_{q}(\mathfrak{g})$-equivariant right $\mathcal{A}$-module $\hat{P}(V \otimes \mathcal{A})$ can be realized on the locally finite part of $\operatorname{Hom}_{\mathbb{C}}\left(M, M_{X_{\lambda}}\right)$ and is isomorphic to locally finite part of $M_{X_{\lambda}} \otimes N$ as a $U_{q}(\mathfrak{g})$ module, for generic $q$. The latter is proved to be isomorphic to subspace of $\mathfrak{k}$-invariants in $\mathbb{C}[G] \otimes X$, thanks to the Peter-Weyl decomposition $U_{q}^{*}(\mathfrak{g})=\sum_{\text {irrep } V} V \otimes V^{*}$ (summation taken is over classes of irreducible finite-dimensional modules). So, $X$ is the fiber of the bundle in the classical limit.

\subsection{Coideal stabilizer subalgebra}

We switch to an alternative realization of quantum vector bundles in terms of quantum symmetric pairs. It is convenient to pass to the left $\mathcal{A}$-module version.

Let $\mathcal{T}$ denote the Hopf dual of $U_{q}(\mathfrak{g})$ and $T=\left(T_{i j}\right)_{i, j=1}^{n+1}$ its matrix of generators. It is invertible with $\left(T^{-1}\right)_{i j}=\gamma\left(T_{i j}\right)$, where $\gamma$ is the antipode of $\mathcal{T}$. One has two commuting left and right translation actions of $U_{q}(\mathfrak{g})$ on $\mathcal{T}$ expressed through the Hopf paring and the comultiplication in $\mathcal{T}$ by

$$
h \triangleright a=a^{(1)}\left(h, a^{(2)}\right), \quad a \triangleleft h=\left(a^{(1)}, h\right) a^{(2)}, \quad a \in \mathcal{T}, \quad h \in U_{q}(\mathfrak{g}) .
$$

They are compatible with multiplication on $\mathcal{T}$ making it a $U_{q}(\mathfrak{g})$-bimodule algebra.

Denote by $\pi: U_{q}(\mathfrak{g}) \rightarrow \operatorname{End}\left(\mathbb{C}^{n+1}\right)$ the natural representation homomorphism. Let $\mathcal{R}$ be a universal R-matrix of $U_{q}(\mathfrak{g})$. The element $\mathcal{R}_{21} \mathcal{R}$ commutes with the coproduct $\Delta(x)$ for all $x \in U_{q}(\mathfrak{g})$. Introduce the matrix $\mathcal{Q}=(\pi \otimes \mathrm{id})\left(\mathcal{R}_{21} \mathcal{R}\right)$ with entries in $U_{q}(\mathfrak{g})$. Denote by $R=(\pi \otimes \pi)(\mathcal{R}) \in \operatorname{End}\left(\mathbb{C}^{2 n+1}\right) \otimes \operatorname{End}\left(\mathbb{C}^{2 n+1}\right)$ the image of $\mathcal{R}$. One can choose $\mathcal{R}$ such that $R$ is proportional to the $R$-matrix used in [FRT]. Following [M2], introduce a matrix 
$A \in \operatorname{End}\left(\mathbb{C}^{2 n+1}\right)$ by

$$
A=\left(\begin{array}{ccccc}
x_{1}+q^{-2} x_{2} & 0 & \cdots & 0 & c \\
0 & q^{-2} x_{2} & 0 & 0 & 0 \\
\vdots & 0 & \ddots & 0 & \vdots \\
0 & 0 & 0 & q^{-2} x_{2} & 0 \\
d & 0 & \cdots & 0 & 0
\end{array}\right)
$$

where $c d=-q^{-2} x_{1} x_{2} \in \mathbb{C} \backslash\{0\}$. It solves the Reflection Equation $[\mathrm{KS}]$

$$
R_{21} A_{1} R_{12} A_{2}=A_{2} R_{21} A_{1} R_{12} \in \operatorname{End}\left(\mathbb{C}^{2 n+1}\right) \otimes \operatorname{End}\left(\mathbb{C}^{2 n+1}\right),
$$

where the indices mark the tensor factors. The assignment $\mathcal{Q} \mapsto T^{-1} A T$ defines an equivariant embedding $\mathcal{A} \subset \mathcal{T}$, where $\mathcal{T}$ is regarded as a $U_{q}(\mathfrak{g})$-module under the left translation action. The matrix $A$ determines a homomorphism $\chi: \mathcal{A} \rightarrow \mathbb{C}, \mathcal{Q}_{i j} \mapsto A_{i j}$. It factors through the composition $\mathcal{A} \rightarrow \mathcal{T} \rightarrow \mathbb{C}$, where the right arrow is the counit $\epsilon$.

The entries of the matrix $(\pi \otimes \mathrm{id})\left(\mathcal{R}_{21}\right) A_{1}(\pi \otimes \mathrm{id})\left(\mathcal{R}_{12}\right) \in \operatorname{End}\left(\mathbb{C}^{2 n+1}\right) \otimes U_{q}(\mathfrak{g})$ generate a left coideal subalgebra $\mathcal{B} \subset U_{q}(\mathfrak{g})$, such that $\mathcal{A}$ is the subalgebra of $\mathcal{B}$-invariants: $a \triangleleft b=\epsilon(b) a$ for all $b \in \mathcal{B}$ and $a \in \mathcal{A}$. The algebra $\mathcal{B}$ is a deformation of $U\left(\mathfrak{k}^{\prime}\right)$, where $\mathfrak{k}^{\prime} \simeq \mathfrak{k}$.

Let $V$ be a finite-dimensional $U_{q}(\mathfrak{g})$-module and $\hat{P} \in V \otimes \mathcal{A}$ an invariant idempotent. In the Sweedler notation, the invariance reads as $\hat{P}_{1} \otimes h \triangleright \hat{P}_{2}=\gamma\left(h^{(1)}\right) \hat{P}_{1} h^{(2)} \otimes \hat{P}_{2}$ for all $h \in U_{q}(\mathfrak{g})$.

Lemma 4.4. The projector $P=\hat{P}_{1} \chi\left(\hat{P}_{2}\right) \in \operatorname{End}(V)$ commutes with $\mathcal{B}$.

Proof. Recall that $\chi$ is the restricted counit $\epsilon$ once $\mathcal{A}$ is realized as a subalgebra in $\mathcal{T}$. For all $b \in \mathcal{B}$ and all $a \in \mathcal{A}$ one has $\epsilon(b \triangleright a)=(b, a)=\varepsilon(a \triangleleft b)=\epsilon(b) \epsilon(a)$. Then $\gamma\left(b^{(1)}\right) P b^{(2)}=$ $\gamma\left(\hat{P}_{1}\right) \epsilon\left(b \triangleright \hat{P}_{2}\right)=\epsilon(b) P$, which implies the lemma.

Proposition 4.5. 1. Every finite-dimensional (right) $U_{q}(\mathfrak{g})$-module $V$ is completely reducible over $\mathcal{B}$ for all $q$ except for a finite set.

2. Each irreducible $\mathcal{B}$-submodule in $V$ is a deformation of a classical $U\left(\mathfrak{k}^{\prime}\right)$-submodule with the same multiplicity.

3. Each $\mathcal{B}$-submodule in $V$ is the image of a $\mathcal{B}$-invariant projector $(\mathrm{id} \otimes \chi)(\hat{P})$, where $\hat{P} \in \operatorname{End}(V) \otimes \mathcal{A}$ is a $U_{q}(\mathfrak{g})$-invariant idempotent. 
Proof. Consider the unit resolution $\operatorname{id}_{V \otimes M}=\sum_{i} \hat{P}_{i}$ by simple invariant idempotents $\hat{P}_{i} \in$ $\operatorname{End}(V \otimes \mathcal{A})$, for generic $q$. Let $P_{i}=\left(\operatorname{id}_{V} \otimes \chi\right)\left(\hat{P}_{i}\right)$ be a projector to a $\mathcal{B}$-submodule in $V$. All $P_{i}$ are rational functions in $q$ and deliver the unit resolution $\operatorname{id}_{V}=\sum_{i} P_{i}$ for all $q$ but from a finite set. This implies that the ranks of $P_{i}$ stay constant where they are defined. In the classical limit, $X_{i}=V P_{i}$ is irreducible over $\mathfrak{k}^{\prime}$, therefore $\mathcal{B}$ fills up entire $\operatorname{End}\left(X_{i}\right)$. That is so for almost all $q$, since image cannot reduce in deformation. Therefore $X_{i}$ is irreducible over $\mathcal{B}$ for all but a finite number of $q$. This proves the first and second statement and readily implies the third since every irreducible submodule is then separable as a direct summand. The corresponding $U_{q}(\mathfrak{g})$-invariant idempotent can be recovered as shown in Lemma 4.6 below.

\subsection{Quantum vector bundles via symmetric pairs}

In this section we realize associated vector bundles as $\mathcal{B}$-invariants in the tensor product of $\mathcal{T}$ and right $\mathcal{B}$-modules. We will work with right modules, which corresponds to the right coset picture.

Thanks to the Peter-Weyl decomposition of $\mathcal{T}$, every finite-dimensional right $U_{q}(\mathfrak{g})$ module $V$ is a left $\mathcal{T}$-comodule. We use a Sweedler-like notation for the left coaction $\delta: V \rightarrow \mathcal{T} \otimes V$, namely $\delta: v \mapsto v^{(1)} \otimes v^{[2]}$. Then $v \triangleleft h=\left(v^{(1)}, h\right) v^{[2]}$ for $v \in V, h \in U_{q}(\mathfrak{g})$.

For each $\mathcal{T}$-comodule $V$, define two $\mathcal{T}$-linear automorphisms of the left $\mathcal{T}$-module $\mathcal{T} \otimes V$,

$$
\iota: a \otimes v \mapsto a v^{(1)} \otimes v^{[2]}, \quad \bar{\imath}: a \otimes v \mapsto a \gamma\left(v^{(1)}\right) \otimes v^{[2]} .
$$

It is easy to check that $\iota \bar{\iota}=\bar{\iota} \iota=\mathrm{id}$ and

$$
\iota(\mathcal{T} \otimes V)^{\mathcal{B}}=\mathcal{A} \otimes V, \quad \bar{\iota}(\mathcal{A} \otimes V)=(\mathcal{T} \otimes V)^{\mathcal{B}} .
$$

We make $V$ a left $U_{q}(\mathfrak{g})$-module via the action $h \bullet v=v \triangleleft \gamma(h), v \in V$, and consider $\mathcal{A} \otimes V$ as a left $U_{q}(\mathfrak{g})$-module. The tensor product $\mathcal{T} \otimes V$ is also a left $U_{q}(\mathfrak{g})$-module with respect to the left translations on $\mathcal{T}$ and the trivial action on $V$. Then the map $\iota$ is $U_{q}(\mathfrak{g})$-invariant, as well as the subspace $\iota(\mathcal{T} \otimes V)^{\mathcal{B}}$.

The algebra $\operatorname{End}(V)$ becomes a natural right comodule whose coaction satisfies the identity $\left(E^{(1)}, h\right) E^{[2]}=\gamma\left(h^{(1)}\right) E h^{(2)}$ for all $E \in \operatorname{End}(V)$ for all $h \in U_{q}(\mathfrak{g})$. Let $\hat{P} \in \operatorname{End}(V) \otimes \mathcal{A}$ be an invariant indecomposable idempotent. If $\left\{E_{i}\right\} \subset \operatorname{End}(V)$ is a basis, then $\hat{P}=\sum_{i} E_{i} \otimes a_{i}$ for some $a_{i} \in \mathcal{A} \subset \mathcal{T}$. The $\mathcal{B}$-invariant projector $P=\sum_{i} E_{i} \epsilon\left(a_{i}\right)$ defines an irreducible right $\mathcal{B}$-submodule $X=V P$. 
Lemma 4.6. One has $\iota(1 \otimes P)=\hat{P}_{21}$ and $\bar{\iota}\left(\hat{P}_{21}\right)=1 \otimes P$.

Proof. Indeed, the $U_{q}(\mathfrak{g})$-invariance of $\hat{P}$ implies $\sum_{i} h \triangleright a_{i} \otimes E_{i}=\sum_{i} a_{i} \otimes E_{i} \triangleleft h$ for all $h \in U_{q}(\mathfrak{g})$. Pairing the $\mathcal{T}$-factor of $\bar{\iota}\left(\hat{P}_{21}\right)$ with arbitrary $h \in U_{q}(\mathfrak{g})$ we get

$$
\sum_{i}\left(a_{i} \gamma\left(E_{i}^{(1)}\right), h\right) \otimes E_{i}^{[2]}=\sum_{i}\left(a_{i}, h^{(1)}\right)\left(\gamma\left(E_{i}^{(1)}\right), h^{(2)}\right) \otimes E_{i}^{[2]}=\sum_{i}\left(a_{i}, h^{(1)}\right) \otimes E_{i} \triangleleft \gamma\left(h^{(2)}\right) .
$$

The last term is

$$
\sum_{i}\left(\gamma\left(h^{(2)}\right) \triangleright a_{i}, h^{(1)}\right) \otimes E_{i}=\sum_{i}\left(a_{i}, h^{(1)} \gamma\left(h^{(2)}\right)\right) \otimes E_{i}=\sum_{i} \epsilon(h) \epsilon\left(a_{i}\right) \otimes E_{i}
$$

due to invariance of $\hat{P}$. This proves the right equality, which implies the left one.

The subspaces $(\mathcal{A} \otimes V) \hat{P}$ and $(\mathcal{T} \otimes V P)^{\mathcal{B}}$ in $\mathcal{T} \otimes V$ are invariant under the left action of $\mathcal{A}$ restricted from $\mathcal{T}$. They are also $U_{q}(\mathfrak{g})$-modules as explained above.

Proposition 4.7. The map $\bar{\iota}:(\mathcal{A} \otimes V) \hat{P}_{21} \rightarrow(\mathcal{T} \otimes V P)^{\mathcal{B}}$ establishes an isomorphism of the left $\mathcal{A}$-modules and $U_{q}(\mathfrak{g})$-modules.

Proof. Indeed, pick up $v \in V$. Then by Lemma 4.6, we get

$$
\bar{\iota}\left((1 \otimes v) \hat{P}_{21}\right)=\sum_{i} a_{i} \gamma\left(E_{i}^{(1)}\right) \gamma\left(v^{(1)}\right) \otimes v^{[2]} E_{i}^{[2]}=\bar{\iota}(1 \otimes v)(1 \otimes P) .
$$

The right formula in $(4.1)$ and $\mathcal{B}$-invariance of $P$ yield $\bar{\iota}(1 \otimes v) P \in(\mathcal{T} \otimes V P)^{\mathcal{B}}$, again by Lemma 4.6. On the other hand,

$$
\iota(1 \otimes v P)=\iota(1 \otimes v) \iota(1 \otimes P)=\iota(1 \otimes v) \hat{P}_{21} \in(\mathcal{A} \otimes V) \hat{P}_{21}
$$

Now $U_{q}(\mathfrak{g})$ - and $\mathcal{A}$-linearity of $\iota$ and $\bar{\iota}$ complete the proof.

Fix an invariant idempotent $\hat{P}$ with the corresponding $\mathcal{B}$-invariant projector $P$ and consider the right $\mathcal{B}$-module $X=V P$.

Theorem 4.8. The $\mathcal{A}$-module $(\mathcal{T} \otimes X)^{\mathcal{B}}$ is a quantization of the vector bundle $\Gamma\left(\mathbb{P}^{n}, X\right)$.

Proof. The map $\iota:(\mathcal{T} \otimes V)^{\mathcal{B}} \rightarrow \mathcal{A} \otimes V$ is an isomorphism of $U_{q}(\mathfrak{g})$-modules. Let $\hat{P} \in$ $\operatorname{End}(V) \otimes \mathcal{A}$ be an invariant projector. The left $U_{q}(\mathfrak{g})$-module $(\mathcal{A} \otimes V) \hat{P}_{21}$ is isomorphic to $\operatorname{Hom}_{\mathbb{C}}\left(M_{X_{\lambda}}, M\right) \simeq \operatorname{Hom}_{U_{q}(\mathfrak{k})}(\mathcal{T}, X)$. Due to the Peter-Weyl decomposition, the latter is a quantization of $(\mathbb{C}[G] \otimes X)^{\mathfrak{k}}$ as required. 


\section{References}

[AM] Ashton, T., Mudrov, A.: Representations of quantum conjugacy classes of orthosymplectic groups, J. Math. Sci. 213 637-650 (2016).

[BFFLS] Bayen, F., Flato, M., Fronsdal, C., Lichnerowicz, A., and Sternheimer, D.: Deformation Theory and Quantization, Ann. Physics, 111 (1978), 61-110.

[ChP] Chari, V. and Pressley, A.: A guide to quantum groups, Cambridge University Press, Cambridge 1994.

[Dr] Drinfeld, V.: Quantum Groups. In Proc. Int. Congress of Mathematicians, Berkeley 1986, Gleason, A. V. (eds) pp. 798-820, AMS, Providence (1987).

[DM1] Donin, J., Mudrov, A.: Dynamical Yang-Baxter equation and quantum vector bundles, Commun. Math. Phys. 254 (2005), 719-760.

[DM2] Donin, J., Mudrov, A.: Method of quantum characters in equivariant quantization, Commun. Math. Phys. 234 (2003), 533-555.

[EV] P. Etingof, A. Varchenko, Dynamical Weyl groups and applications, Adv. Math. 167 (2002), 74-127.

[FRT] Faddeev, L., Reshetikhin, N., and Takhtajan, L.: Quantization of Lie groups and Lie algebras, Leningrad Math. J., 1 (1990), 193-226.

[Kolb] Kolb, S.:Quantum symmetric Kac-Moody pairs, Adv. Math. 267 (2014), 395-469,

[KO] Khoroshkin, S. M., and Ogievetsky, O.: Mickelsson algebras and Zhelobenko operators, J. Alg. 319 (2008), 2113-2165.

[KST] Karolinsky, E., Stolin, A., Tarasov, V.: Equivariant quantization of Poisson homogeneous spaces and Kostant's problem, J. Alg. 409 (2014), 362-381.

[KS] Kulish, P. P., Sklyanin, E. K.: Algebraic structure related to the reflection equation, J. Phys. A, 25 (1992), 5963-5975.

[Let] Letzter, G.: Symmetric pairs for quantized enveloping algebras, J. Algebra 220 (1999), no. 2, 729-767. 
[Mol] Molev, A.: Gelfand-Tsetlin bases for classical Lie algebras, in: "Handbook of Algebra", Vol. 4 (M. Hazewinkel, Ed.) 2006, pp. 109-170.

[M1] Mudrov, A.: Quantum conjugacy classes of simple matrix groups, Commun. Math. Phys., 272 (2007), 635 - 660.

[M2] Mudrov, A.: Characters of the $U_{q}(s l(n))$-reflection equation algebra, Lett. Math. Phys., 60 (2002), \# 3, 283-291.

[M3] Mudrov, A.: Contravariant form on tensor product of highest weight modules, preprint arXiv:1709.08394.

[STS] Semenov-Tian-Shansky, M.: Poisson-Lie Groups. Quantum Duality Principle, and the Quantum Double. Contemp. Math., 175 (1994), 219-248.

[S] Serre, J.-P.: Faisceaux Algebriques Coherents, Ann. Math., 61 \# 2 (1955), 197-278.

[Sw] Swan, R.: Vector Bundles and Projective Modules, Trans. AMS, 105 \# 2 (1962), 264277. 